\title{
Severe Dehiscence of Gastrojejunal Anastomosis after Gastric Bypass: Its Cure by Using Partially Covered Stent and Avoiding Migration
}

\author{
Helga C. A. W. Alhinho ${ }^{1}$ - Flávio C. Ferreira ${ }^{1} \cdot$ Rodrigo C. L. Medeiros ${ }^{1}$ • \\ Álvaro A. B. Ferraz ${ }^{1} \cdot$ Josemberg M. Campos ${ }^{1}$
}

Published online: 27 November 2017

(C) Springer Science+Business Media, LLC, part of Springer Nature 2017

\begin{abstract}
Background Gastric leak is a severe complication of gastric bypass that is associated with significant morbidity and mortality. Anastomosis dehiscence usually occurs at gastrojejunal anastomosis and can appear simultaneously with gastric leak, for which treatment can be a challenge. Fistula may have several clinical impacts, depending on patient-related factors, fistula characteristics, onset time, and therapy proposal. Abdominal toilet, drainage, gastrostomy, and revisional surgery constitute the traditional approaches to dehiscence and fistula closure, with variable results. Currently, endoscopic stents are gaining space, promoting fistula sealing, secretion deviation, treating gastric stricture, and allowing early oral diet. Herein, we present a case of severe gastrojejunal anastomosis dehiscence treated with partially covered stent.

Materials and Methods We present a video of a 39-year-old man with a body mass index of $40 \mathrm{Kg} / \mathrm{m}^{2}$ who underwent a Roux-en-Y gastric bypass and presented fever and leukocytosis. Gastric leak was diagnosed 7 days after the bariatric surgery. At first, he was submitted to three reoperations: laparotomy with abdominal toilet, abdominal drain, and gastrostomy. Sepsis was controlled, but drain output maintained the same debit. On the 22nd POD, it was decided to place a metallic stent. As the first step, an endoscopist looked at the lesser curvature. There was no continuity to the alimentary limb, and the anastomosis was disrupted. Careful inflation and washing was done, allowing identification of the alimentary limb, followed by guidewire passage, with radioscopic control. Once the guidewire was positioned, stent placement was possible and safe. Upper edge of stent was placed in the lower third of the esophagus. Results Patient progressed uneventfully. After 4 weeks, stent removal was attempted. However, it was not possible due to endoluminal tissue hyperplasia. Argon plasma was used three times to promote proliferative mucosa ablation. Stent was removed after 53 days, with no migration. The abdominal drain was removed 1 week later. After 6-months follow-up, the patient remains asymptomatic.

Conclusion Early dehiscence closure was observed, without recurrence. The use of partially covered self-expandable metallic stent is associated with lower migration rates; however, removal can be technically difficult due to tissue hyperplasia.
\end{abstract}

Keywords Gastric bypass · Leak · Anastomosis dehiscence $\cdot$ Endoscopic stents $\cdot$ Bariatric endoscopy

Electronic supplementary material The online version of this article (https://doi.org/10.1007/s11695-017-3016-8) contains supplementary material, which is available to authorized users.

Josemberg M. Campos

josembergcampos@gmail.com

1 Universidade Federal de Pernambuco, Recife, Brazil
Compliance with Ethical Standards

Conflict of Interest The authors declare that they have no conflicts of interest.

Ethical Approval All procedures performed in studies involving human participants were in accordance with the ethical standards of the institutional and/or national research committee and with the 1964 Helsinki declaration and its later amendments or comparable ethical standards.

Informed Consent Informed consent was obtained from the individual participant included in the study. 